\title{
A Study on Prediction of Birth Weight for Infants using Transition Probability Matrix with respect to Haemoglobin Count
}

\author{
M. Helen Sheeba ${ }^{1}$ and Dr. S. Durai Raj ${ }^{2}$ \\ ${ }^{1}$ Assistant Professor in Mathematics, \\ Pioneer Kumaraswamy College, Nagercoil, \\ Affiliated to Manonmaniam Sundaranar University, Tirunelveli - 627 012, Tamil Nadu, India, \\ Email: hsheeba67@gmail.com \\ ${ }^{2}$ Principal, Pioneer Kumaraswamy College, Nagercoil, \\ Affiliated to Manonmaniam Sundaranar University, Tirunelveli - 627 012, Tamil Nadu, India, \\ Email: durairajsprincpkc@gmail.com
}

\begin{abstract}
Rapid developments in stochastic models in recent years have resulted in significant changes in the way the world operates. This has an impact on educational needs, both in terms of the content and the delivery of educational services and there has been increasing pressure on decision - makers to acquire new technologies. This paper examines the mature and premature birth using Haemoglobin count during pregnancy time by Transition Probability Matrix. The maternal variables such as maternal weight, maternal height, haemoglobin count and birth weight of babies are considered as important factors in the study of demographic and public health related issues. The result shows that the birth weight mainly depends on Haemoglobin concentration.
\end{abstract}

Keywords- Stochastic models, Transition Probability Matrix, Haemoglobin

\section{INTRODUCTION}

The aim of this paper is to analyze the mature and premature birth using Haemoglobin in the protein molecule in red blood cells that carries oxygen from the lungs to the body's tissues and returns carbon dioxide from the tissues back to the lungs by Transition Probability Matrix. Haemoglobin is made up of four protein molecules that are connected together. The normal ranges for haemoglobin depend on the age and beginning in adolescene, the gender of the person. The normal ranges of haemoglobinare: (i) Newborns: 17 to $22 \mathrm{gm} / \mathrm{dLe}$ (ii) $1^{\text {st }}$ week of age: 15 to $20 \mathrm{gm} / \mathrm{dL}$ (iii) One month of age: 11 to $15 \mathrm{gm} / \mathrm{dL}$ (iv) children: 11 to $13 \mathrm{gm} / \mathrm{dL}$ (v) Adult males: 14 to $18 \mathrm{gm} / \mathrm{dL}$ (vi) Adult women: 12 to $16 \mathrm{gm} / \mathrm{dL}$ (vii) Men after middle age: 12.4 to $14.9 \mathrm{gm} / \mathrm{dL}$ and (viii) Women after middle age: 11.7 to $13.8 \mathrm{gm} / \mathrm{dL}$.

\section{MARKOV CHAIN}

The stochastic process $\left\{X_{n}=n=1,2, \ldots\right\}$ is called a Markov chain, if, for $\mathrm{j}, \mathrm{k}, \mathrm{j}_{1}, \ldots, \mathrm{j}_{\mathrm{n}-1} \in \mathrm{N}$ (or any subset of I),

$$
\begin{aligned}
& \operatorname{Pr}\left\{X_{n}=k / X_{n-1}=j, X_{n-2}=j_{1}, \ldots X_{0}=j_{n-1}\right\} \\
& =\operatorname{Pr}\left\{X_{n}=k / X_{n-1}=j\right\} \\
& =P_{j k} \text { (say) }
\end{aligned}
$$

whenever the first member is defined.

\section{TRANSITION PROBABILITY MATRIX}

The transition probabilities $\mathrm{P}_{\mathrm{jk}}$ satisfy $\sum_{K} P_{j k}=1$ for all j. These probabilities may be written in the matrix form

$$
\mathrm{P}=\left[\begin{array}{cccc}
P_{11} & P_{12} & P_{13} & \cdots \\
P_{21} & P_{22} & P_{23} & \cdots \\
\cdots & \cdots & \cdots & \cdots
\end{array}\right]
$$

This is called the Transition Probability Matrix (or) Transition Probabilities of the Markov Chain. $\mathrm{P}$ is a stochastic matrix.

\section{MODEL DESCRIPTION}

Markov chain is a discrete-time process the process can start in one of these states and move and another state. Each move is called a step. Each step has a probability of its own. If the chain is currently in state $\mathrm{S}_{\mathrm{i}}$, then it moves to state $\mathrm{S}_{\mathrm{j}}$ at the next step with a probability shown by $\mathrm{P}_{\mathrm{ij}}$ and this probability does not depend upon which states the chain was in before the current. The probabilities $\mathrm{P}_{\mathrm{ij}}$ are called transition probabilities. The probabilities can be shown in a matrix called transition matrix. In the $\mathrm{P}$ transition matrix below $P_{i j}$ is the probability of being in state $S_{i}$ at step $n+1$ given that the process was in state $S_{j}$ at step $n$. 


\section{Available online at www.ijrat.org}

A state $\mathrm{S}_{\mathrm{i}}$ of a Markov chain is called an absorbing state if, once the Markov chains enter the state, it is impossible to leave that state would be zero and it is shown as $\mathrm{P}_{\mathrm{ii}}=1$. A Markov chain is absorbing if it has at least one absorbing state and if from every state it is possible to go to an absorbing is called transient. In the hypothetical example below, 0 and 4 are in the absorbing state with the probability of 1 . Therefore the chain is an absorbing chain. States 1, 2 and 3 are transient.

Transition matrix of an absorbing Markov chain follows a canonical form, which means that the transient states come first:

$$
\begin{array}{ccc} 
& A B S & T R \\
P=A B S & I & 0 \\
T R & R & Q
\end{array}
$$

If we have $\mathrm{t}$ transient states and $\mathrm{r}$ absorbent states, then $\mathrm{I}$ : is an r-by-r identity matrix, 0 : is r-by-t zero matrix. R: is a nonzero t-by-r matrix, giving transition probabilities from transient to absorbing states, $\mathrm{Q}$ : is a t-by-t matrix, giving transition probabilities from transient to transient states.

\section{Analysis}

Make a Transition probability matrix using the following states: State 1 is <10grams per deciliter, state 2 is $10-11$ grams per deciliter, state 3 is $11-12$ grams per deciliter, state 4 is $>12$ grams per deciliter, state 5 is premature birth and state 6 is mature birth.

$P=\left[\begin{array}{cccccc}0.1538 & 0.3077 & 0.2308 & 0.2308 & 0.0769 & 0 \\ 0 & 0.125 & 0.25 & 0.25 & 0.375 & 0 \\ 0 & 0 & 0.1667 & 0.3333 & 0.5 & 0 \\ 0 & 0 & 0 & 0 & 1 & 0 \\ 0 & 0 & 0 & 0 & 1 & 0 \\ 0 & 0 & 0 & 0 & 0 & 1\end{array}\right]$

The canonical form of the transition probability matrix is

$$
\mathrm{P}=\left[\begin{array}{cccccc}
1 & 0 & 0 & 0 & 0 & 0 \\
0 & 1 & 0 & 0 & 0 & 0 \\
0 & 0.0769 & 0.1538 & 0.3077 & 0.2308 & 0.2308 \\
0 & 0.375 & 0 & 0.125 & 0.25 & 0.25 \\
0 & 0.5 & 0 & 0 & 0.1667 & 0.3333 \\
0.4375 & 0.125 & 0 & 0 & 0 & 0.5
\end{array}\right]
$$

The matrix is smaller than usual, however, this does not change the process, we can find the $\mathrm{Q}, \mathrm{R}, \mathrm{I}$ and 0 matrices.

$$
\begin{aligned}
& R=\left[\begin{array}{cc}
0 & 0.0769 \\
0 & 0.375 \\
0 & 0.5 \\
0.4375 & 0.125
\end{array}\right] Q=\left[\begin{array}{cccc}
0.1538 & 0.3077 & 0.2308 & 0.2308 \\
0 & 0.125 & 0.25 & 0.25 \\
0 & 0 & 0.1667 & 0.333 \\
0 & 0 & 0 & 0.5
\end{array}\right] \\
& I=\left(\begin{array}{ll}
1 & 0 \\
0 & 1
\end{array}\right) \& 0=\left(\begin{array}{llll}
0 & 0 & 0 & 0 \\
0 & 0 & 0 & 0
\end{array}\right)
\end{aligned}
$$

We can subtract the "Q" matrix from the "I" matrix and then take the inverse of that matrix using MAT Lab.

$\mathrm{N}=(1-\mathrm{Q})^{-1}=\left[\begin{array}{cccc}1.1818 & 0.4156 & 0.4520 & 1.0546 \\ 0 & 1.1429 & 0.3429 & 0.8 \\ 0 & 0 & 1.2 & 0.8 \\ 0 & 0 & 0 & 2\end{array}\right]$

The probability that an absorbing chain will be absorbed in the absorbing state since we are only interested in the time that the machine will spend in that transient period using MAT Lab.

$\mathrm{B}=\mathrm{N} * \mathrm{R}=\left[\begin{array}{cc}0.4614 & 0.6045 \\ 0.35 & 0.7 \\ 0.35 & 0.7 \\ 0.8750 & 0.25\end{array}\right]$

The above values reveal that the expected probability that someone who have $<10$ grams per deciliter in haemoglobin will eventually result in premature birth is 0.6045 and mature birth is 0.4614 only. $10-11$ grams per deciliter result in premature birth is 0.7 and mature is 0.35 and those who have more than 12 grams per deciliter will result in eventually mature is 0.8750 and premature is 0.25 .

\section{CONCLUSION}

From this study, it is observed that the birth mainly depends on Hemoglobin concentration. Hence a mother with high Hemoglobin concentration can avoid low birth weight. So a pregnant woman should intake additional nutritional food to increase the Haemoglobin concentration and to avoid the health risk problems among the neonates. Some reasons for the case of low birth weight are that the mother has not obtained the appropriate nutrition, early marriage, late pregnancy at around 35 years, mother below 40 Kilograms, mother has anaemic problems, small placenta, chronic placenta. Lack of oxygen also leads to low birth weight and it also happens due to mother's hypertension and malnutrition. Some actions 


\section{Available online at www.ijrat.org}

have to be taken before giving birth to avoid low birth weight infants. The mother has to take up regular check up, appropriate nutrition intake, check the haemoglobin status and take iron supplements.

\section{REFERENCES}

[1] Arbuckle T.E and Sherman GJ, Comparison of the risk factors for preterms delivery and intrauterine growth retardation, Peachtree Perinat, Epidemol, (1989) Vol. 3, pp. 115-129.

[2] Bhatia B.D and Tyagi N.K., Birth weight relationship with other Foelat Anthrometric parameter Indian pediatrics (1984), Vol. 21, pp. 833-838.

[3] Philip Steer U Kondaveeli C Bary-Kinsella, Relation between maternal haemoglobin concentration and birth weight in different ethnic groups. Biomedical journal. (1995), 310(1), pp. 489-491.

[4] Warren Ewens, "Mathematical Population Genetics", Second edition, 2004, pp. 86-91, 9299, 104-109.

[5] Nagarajan. D, Sunitha. P, Helen Sheeba. M, Nagarajan, V Statistical Analysis of Blood Group using Maximum Likelihood estimation, International Journal of Engineering and Technical Research 2014, Vol. 2, Issue 6, pp. 6870.

[6] J. Medhi. Stochastic processes and its applications. 